\title{
Sex differences in cardiovascular control by nitric oxide in normotensive and hypertensive rats
}

\author{
Oxana Semyachkina-Glushkovskaya*, Tatyana Anishchenko, Sergey Kapralov, \\ Roman Novikov, Konstantin Skvorcov, Yana Kuznecova, Antonina Kuznecova
}

Saratov State University, Saratov, Russia; *Corresponding Author: glushkovskaya@mail.ru

Received 21 November 2009; revised 7 January 2010; accepted 9 January 2010.

\begin{abstract}
$R$ Nitric oxide probably plays an important role in mechanisms determining sexual dimorphism in the development of cardiovascular diseases, including hypertension. Because stress together with gender are significant cardiovascular risk factors, we studied the role nitric oxide in cardiovascular regulation in male and female normotensive and hypertensive rats under normal and stress conditions. Experiments were performed in mongrel normotensive and hypertensive (two kidney, one clip) rats of both sexes, weighing $200-250 \mathrm{~g}$. The study of mean arterial pressure and heart rate was carried out: 1) under control condition; 2) during nitric oxide blo- ckade by $N^{G}$-nitro-L-arginine-methyl ester (L-NAME, 10 $\mathrm{mg} / \mathrm{kg}$, iv) 3) during $60 \mathrm{~min}$ immobilization stress and recovery; 4) during $60 \mathrm{~min}$ immobilization stress + L-NAME and recovery. We found that the severity of hypertension in females was lower than in males. We also observed that both normotensive and hypertensive females demonstrated more favorable pattern of cardiovascular responses to stress. At rest, nitric oxide blockade increased the mean arterial pressure and decreased the heart rate more effectively in female normotensive and hypertensive rats than in male groups. During stress, nitric oxide blockade modified the stressinduced cardiovascular responses more significantly in female normotensive and hypertensive rats compared with male groups. Our data show that both normotensive and hypertensive females demonstrated the more effective nitric oxide control of cardiovascular activity under normal and especially stress conditions than male groups. This male-female difference may be important mechanism responsible for greater in females vs. males of cardio-
\end{abstract}

vascular resistance to stress and development of hypertension.

Keywords: Nitric Oxide; Stress; Cardiovascular Stress-Reactivity; Sex-Differences

\section{INTRODUCTION}

The clinical and experimental studies show that nitric oxide (NO) plays an important role in regulation of the cardiovascular homeostasis. So, NO inhibits platelet aggregation and adhesion [1], prevents neointimal plaque progression [2], NO is also involved in the baroreflex mechanism [3]; increases cholinergic [4] but inhibits sympathetic influences on the heart [5]. NO deficiency contributes to the pathogenesis of different cardiovascular diseases including hypertension [6,7] and NO donors used in cardiovascular therapeutics [8].

It is known that incidence of cardiovascular diseases such as hypertension is significantly lower in women of reproductive age compared with age-matched men. Although the mechanisms underlying this "cardioprotection" of females are not fully elucidated, the enhanced vascular production of NO has been shown to have a potential role in both humans and rats. In women, whole body NO production and NO-dependent vasodilatation is greater than in men $[9,10]$. In vitro experiments have demonstrated that the aorta, as well as coronary, cerebral and skeletal muscle arteries from female rats appear to produce a greater amount of NO compared with those from males [11-14].

In our studies we observed that female compared with male rats demonstrate higher basal and stressed levels of NO [15] that is associated with more favorable pattern of cardiovascular responses to stress in females vs. males [16]. This fact may be due to cardioprotective role of NO during stress. Indeed in some investigations it has been reported that NO possesses stress-limiting activity [17] and produces a regional vasodilatatory effect [18]. 
Thus, an enhanced function of the NO system in females may contribute to a lower incidence of vascular disease. Despite the well-documented gender-differences in the development and severity of hypertension in humans and in animal models, as well as the increasing evidence supporting the cardioprotective role of NO during stress and cardiovascular pathology, the gender particularities in activity of the NO-ergic system during cardiovascular dysfunction and stress remain little studied.

\section{METHODS}

\subsection{Animals}

Experiments were performed in mongrel normotensive (NT) and hypertensive (HT) rats of both sexes, weighing 200 to $250 \mathrm{~g}$. All procedures were performed in accordance with the Guide for the Care and Use of Laboratory Animals published by the US National Institutes of Health (NIH Publication No. 85-23, revised 1996).

\subsection{Surgical Preparation}

To induce two kidney, one clip (2K1C) hypertension rats were clipped at the left renal artery with a silver clip having an inner gap of $0.25 \mathrm{~mm}$ under anesthesia with ketamine $(40 \mathrm{mg} / \mathrm{kg}$, ip). Seven weeks after clipping hypertensive rats were used in all sets of experiment.

The day before the experiment, both NT and HT rats were instrumented with polyethylene catheters for monitoring mean arterial pressure (MAP) and heart rate (HR). For implantation of catheters, rats were anesthetized with ketamine $(40 \mathrm{mg} / \mathrm{kg}$, ip) supplemented with xylazine $(5 \mathrm{mg} / \mathrm{kg}$, ip). Polyethylene catheter (PE-50 with a PE-10 tip, Scientific Commodities INC., Lake Havasu City, Arizona) was inserted into the left common carotid artery. In addition, the left femoral vein was catheterized with PE-50 tubing fused PE-10 for drug infusion.

\subsection{Experimental Procedure}

HR and MAP were recorded in the home cages of conscious, unrestrained rats. Blood pressure signals were acquired on PowerLab system (ADInstruments, Australia) using pressure transducer. The control group included intact, but not sham-operated animals since we found that the basal and stressed values of MAP and HR didn't differ significantly between sham-clipped rats and NT (nonclipped) group or between sexes within each group and also the blood corticosterone level returned to normal 1 day after surgery (laparotomy) [19].

Protocol 1: Influences of immobilization stress (IS) on cardiovascular activity in NT and HT male and female rats. After base-line measurement, HR and MAP were recorded continuously during $60 \mathrm{~min}$ immobilization and 60-min recovery. This protocol was administered to a group of NT male $(\mathrm{n}=10)$, female $(\mathrm{n}=10)$ and to a group of HT male $(n=10)$, female $(n=10)$ rats.

Protocol 2: Effect of NO synthesis inhibition on MAP and HR in NT and HT rats of both sexes at rest. After base-line measurement, changes in hemodynamic responses to inhibition of NO synthesis were evaluated by intravenous administration of $\mathrm{N}^{\mathrm{G}}$-nitro-L-arginine methyl ester (L-NAME, Sigma Chemical Co, $10 \mathrm{mg} / \mathrm{kg}$, iv). HR and MAP were recorded continuously during 120 min after L-NAME injection. This protocol was administered to a group of NT male $(\mathrm{n}=12)$, female $(\mathrm{n}$ $=15)$ and to a group of HT male $(\mathrm{n}=12)$, female $(\mathrm{n}=12)$ rats.

Protocol 3: Effect of NO synthesis inhibition on MAP and HR in NT and HT rats of both sexes under IS condition. After base-line measurement, male and female rats of both groups were treated L-NAME. Then rats were subjected to 60-min immobilization. HR and MAP were recorded continuously during $60 \mathrm{~min}$ IS and 60-min recovery. This protocol was administered to a group of normotensive male $(\mathrm{n}=10)$, female $(\mathrm{n}=10)$ and to a group of hypertensive male $(\mathrm{n}=10)$, female ( $\mathrm{n}$ $=10)$ rats.

After performance of all experiments the animals were killed with an intravenous overdose of ketamine $(150 \mathrm{mg} / \mathrm{kg})$. The kidneys were then removed and weighed.

Results were reported as mean \pm standard error of the mean (SEM). The changes in MAP and HR were expressed as percentage changes from control values. The differences from the initial level in the same group were evaluated by Wilcoxon test. Inter-group differences were evaluated using Mann-Whitney test and ANOVA-2 (post hoc analysis with Duncan's rank test). Significance levels were set at $\mathrm{p}<0.05$ for all analyses.

\section{RESULTS}

The development of renovascular hypertension (twokidney, one clip, 2K1C) observed 7 weeks after clipping of left renal artery became more severe in males vs. females. So, in all sets of experiment the baseline MAP values were significantly higher in HT males relative to HT females (Table 1). Furthermore, the weight of clipped kidneys in males and females decreased by 10, 6 $(\mathrm{p}<0.05)$ and 6, $2(0.05)$ times, respectively.

\subsection{The Hemodynamic Effects of is in Normotensive and Hypertensive Male and Female Rats}

The IS caused the significant tachycardia and increase in 
Table 1. The baseline mean arterial pressure (MAP, $\mathrm{mmHg}$ ) and heart rate (HR, beats/min) values in normotensive and hypertensive male and female rats.

\begin{tabular}{|c|c|c|c|c|c|c|c|c|}
\hline \multirow{3}{*}{ Sets of experiments } & \multicolumn{4}{|c|}{ Normotensive } & \multicolumn{4}{|c|}{ Hypertensive } \\
\hline & \multicolumn{2}{|c|}{ Female } & \multicolumn{2}{|c|}{ Male } & \multicolumn{2}{|c|}{ Female } & \multicolumn{2}{|c|}{ Male } \\
\hline & MAP & HR & MAP & HR & MAP & HR & MAP & HR \\
\hline $\begin{array}{l}\text { 1. immobilization } \\
\text { stress } \\
\text { 2. NO blockade with }\end{array}$ & $102 \pm 2$ & $349 \pm 4 \uparrow$ & $105 \pm 3$ & $371 \pm 4$ & $118 \pm 4 \dagger 口$ & $354 \pm 10$ & $140 \pm 3 \square$ & $362 \pm 7$ \\
\hline $\begin{array}{l}\text { L-NAME injection at } \\
\text { rest }\end{array}$ & $101 \pm 2$ & $361 \pm 7$ & $103 \pm 3$ & $358 \pm 6$ & $131 \pm 2 \dagger \square$ & $370 \pm 11$ & $148 \pm 3 \square$ & $377 \pm 9$ \\
\hline $\begin{array}{l}\text { 3. NO blockade with } \\
\text { L-NAME injection } \\
\text { under immobilization } \\
\text { stress }\end{array}$ & $102 \pm 3$ & $353 \pm 5$ & $105 \pm 3$ & $365 \pm 6$ & $137 \pm 3 \dagger \square$ & $367 \pm 8 \square$ & $151 \pm 4 \square$ & $376 \pm 10$ \\
\hline
\end{tabular}

$\square-\mathrm{p}<0.05$ vs. normotensive rats; $\uparrow-\mathrm{p}<0.05$ vs. males

MAP in both NT and HT rats. The effects of IS on cardiovascular activity were different in females and males of both groups.

In NT females the HR increased more significantly $(22-32 \%$ vs. $13-24 \%, p<0.05)$ and recovered more rapidly than in NT males (Figure 1). The basal values of HR were lower in NT females vs. NT males (Table 1). Important notice that despite more severe tachycardia under IS in NT females, the amplitude and duration of MAP elevation were lower than in NT males (Figure 1). Moreover, only in NT females, short-lasting stress-induced MAP increase was followed by transient hypotension. There was no significant sex difference in the basal MAP values in NT group (Table 1).

\subsection{Hypertension Versus Normotension}

In HT rats in comparison with NT ones, cardiac and vascular responses to IS were decreased in females but increased in males (Figures 1 and 2). So, in females, hypertension was accompanied by decrease in stressevoked tachycardia in comparison with NT females: in

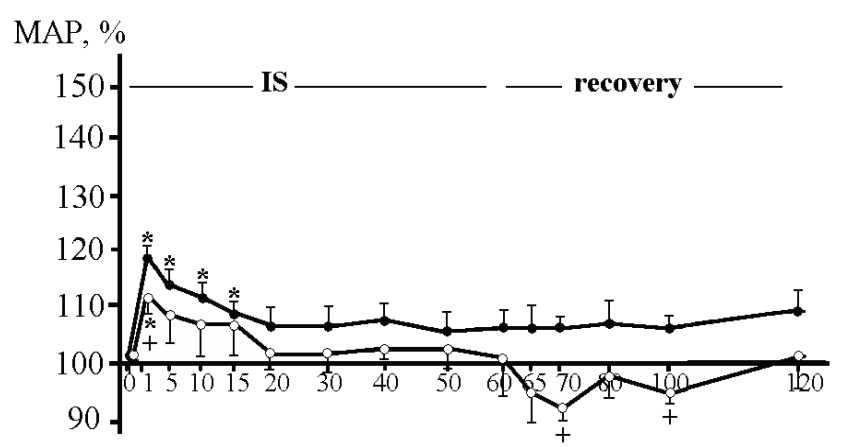

Time, $\min$

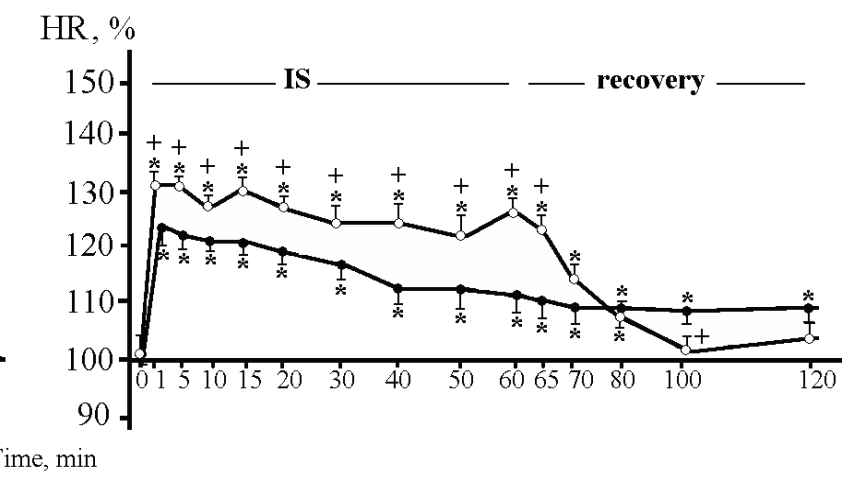

(a)
HT females during IS the HR increased by only $10-16 \%$ $(\mathrm{p}<0.05)$, while in NT females this parameter increased by $22-32 \%$ ( $p<0.05)$. In HT females, HR returned to normal $5 \mathrm{~min}$ after IS, while in NT females it returned to normal $40 \mathrm{~min}$ after the end of IS. The pressor responses in HT and NT females observed at $1 \mathrm{~min}$ of IS and did not differ by the amplitude. However, in HT females the significant MAP increase was replaced by long-lasting (more than $120 \mathrm{~min}$ ) compensatory hypotensive state.

In contrast to HT females, in males hypertension was accompanied by an increase in cardiac and vascular reactivity to IS. Really, the positive chronotropic effect of IS was significantly higher in HT males $(28-21 \%$, p < $0.05)$ than in NT males $(24-13 \%, \mathrm{p}<0.05)$ over the entire stress period. As for MAP, despite the same of amplitude of stress-induced increase in MAP in both groups the pressor reactions were more prolonged in HT males than in NT males: in NT males the elevated MAP levels were observed during $15 \mathrm{~min}$ of IS, in HT males - during $40 \mathrm{~min}$ of IS and it remained tendentionally higher at the end of IS.

Figure 1. The changes of mean arterial pressure (MAP, $\%-a)$ and heart rate $(\mathrm{HR}, \%-b)$ in normotensive male and female rats during immobilization stress and recovery period. Here and in Figures 2-6: $0 \longrightarrow 0-$ females, $\longrightarrow \longrightarrow-$ males; $p<0.05: *-$ vs. basal level; †-vs. males. 
The decrease in the chronotropic component of IS in HT females and its increase in HT males inverted the sex-related difference in the degree of stress-induced tachycardia (Figure 2). In HT rats, in contrast NT animals, the HR response to IS was more pronounced in males vs. females $(28 \%$ vs. $16 \%, \mathrm{p}<0.05)$. Notice that basal values of HR didn't differ reliably between sexes in HT group.

\subsection{The Hemodynamic Effects of No Blockade in Normotensive and Hypertensive Male and Female Rats at Rest and During Stress}

In both NT and HT rats, NO blockade with L-NAME caused an increase in MAP with a decrease in HR and these changes depended upon the gender and the state of rat.

Female NT rats in comparison with male ones were more sensitive to NO blockade with L-NAME under normal and especially stress conditions. Actually, at rest, L-NAME induced a greater MAP increase with a greater HR decrease in females than in males $(25 \%$ vs. $17 \%, p<$
0,05 for MAP; $18 \%$ vs. $10 \%$, p $0<0,05$ for HR). This sex difference in cardiovascular response to L-NAME was occurred within $30 \mathrm{~min}$ after L-NAME injection (Figure 3). There were no statistical differences in baseline MAP and HR values between NT females and NT males (Table 1).

Cardiovascular responses to IS have been significantly modified by L-NAME in NT males and especially in NT females (Figures 1 and 4). Indeed, IS against NO blockade induced a higher elevation in MAP than IS alone did (34\% vs. $18 \%$, p < 0.05 in NT males and $57 \%$ vs. $11 \%$, p $<0.05$ in NT females). As a result, sex difference in vascular responses to IS was greater in the L-NAME treated group $(57 \%$ vs. $34 \%, \mathrm{p}<0.01)$ than in untreated one $(18 \%$ vs. $11 \%, \mathrm{p}<0.05)$ and observed during $60 \mathrm{~min}$ of IS and $40 \mathrm{~min}$ of recovery period. In both sexes, especially in NT females, stress-evoked MAP elevation was much more continued when compared with IS alone. Actually, the elevated MAP occurred within $60 \mathrm{~min}$ of IS and the MAP didn't reach the basal values even at 60 min after stress-off.
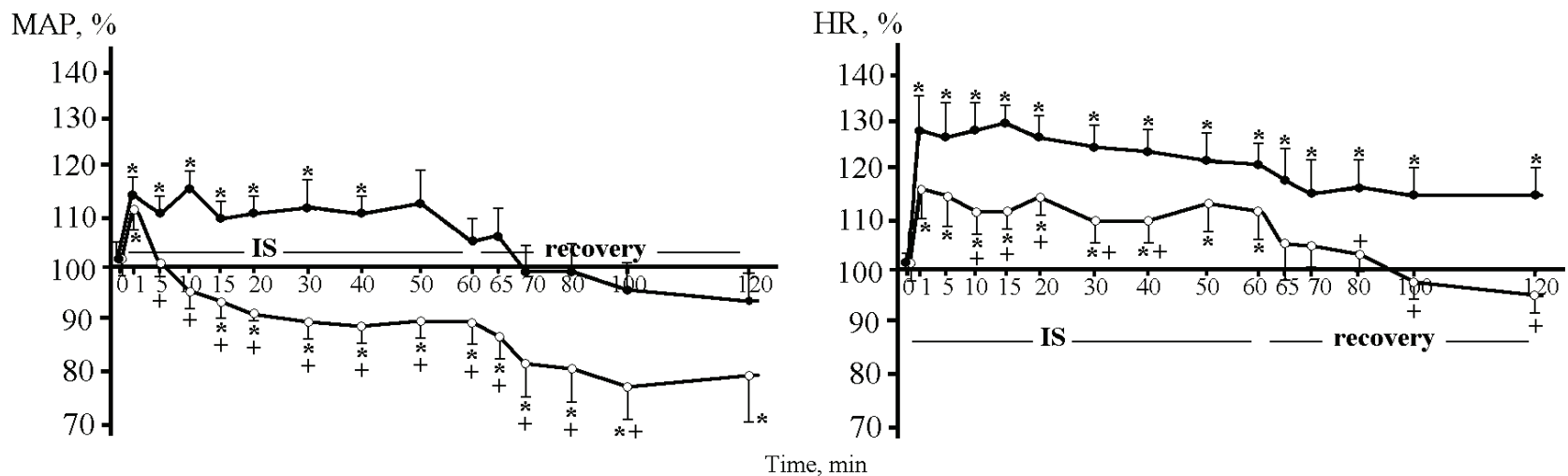

(a) (b)

Figure 2. The changes of mean arterial pressure (MAP, $\%-a)$ and heart rate $(\mathrm{HR}, \%-b)$ in hypertensive male and female rats during immobilization stress and recovery period.

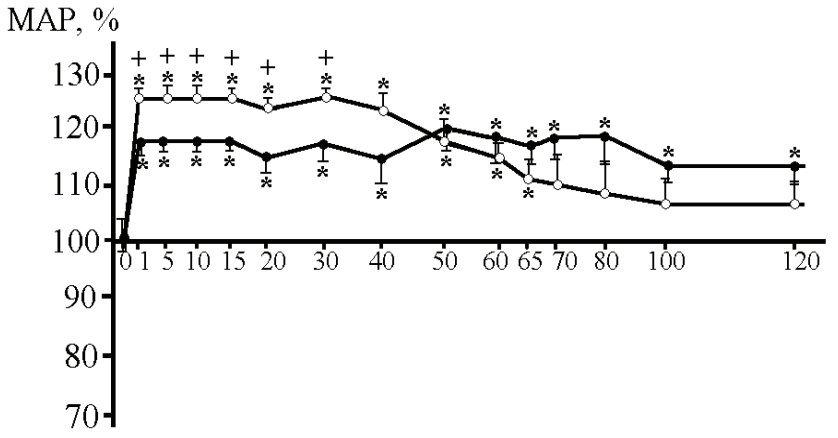

(a)

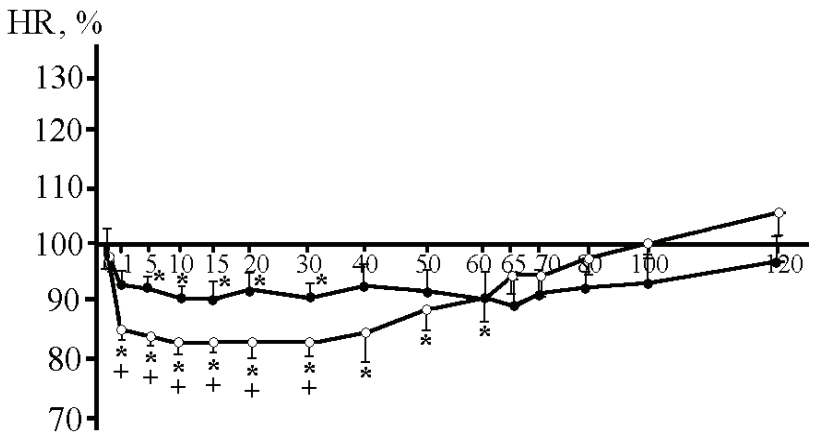

Time, $\min$

(b)

Figure 3. The effects of NO synthesis inhibition by L-NAME on mean arterial pressure (MAP, $\%-a)$ and heart rate (HR, $\%-b)$ in normotensive male and female rats at rest. 


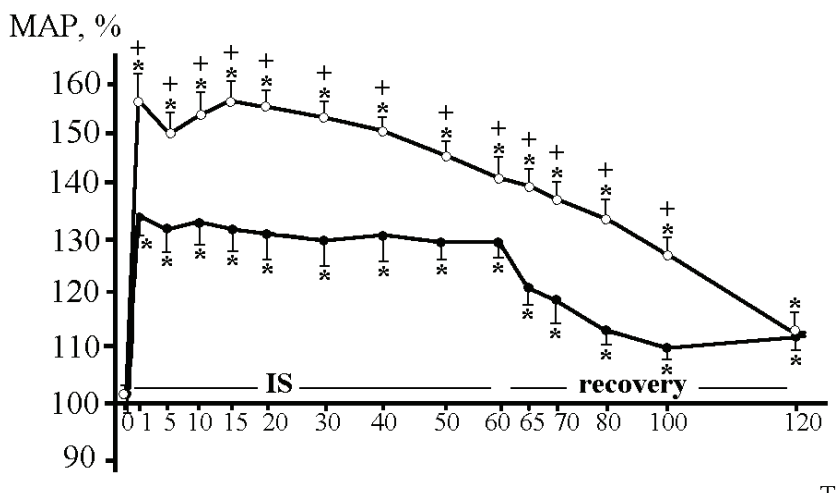

(a)

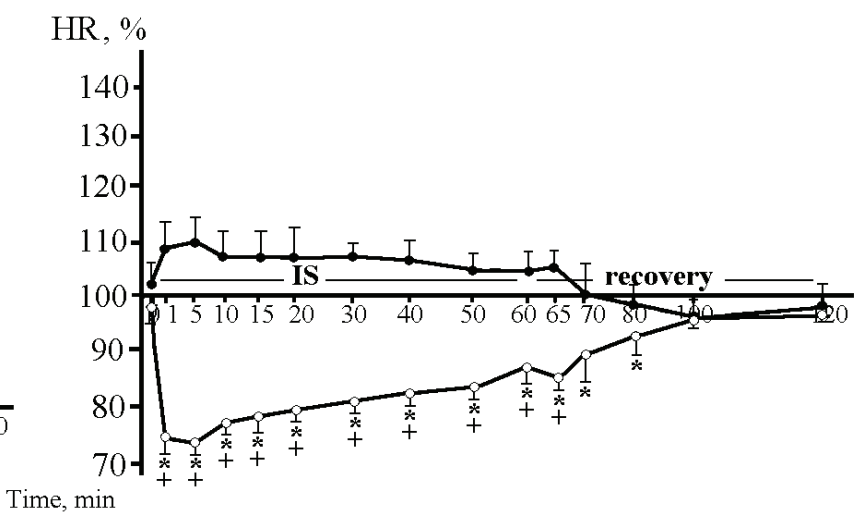

(b)

Figure 4. The effects of NO synthesis inhibition by L-NAME on mean arterial pressure (MAP, $\%-a)$ and heart rate (HR, \% - b) in normotensive male and female rats under stress condition.

Cardiac effects of IS were also modified by L-NAME in NT males and especially in NT females (Figures 1 and 4). Actually, in NT males, tachycardic response to IS was significantly suppressed by L-NAME. In NT females, tachycardic effects of IS were abolished completely and replaced by bradycardic effects of L-NAME, that were more pronounced in stressed females compared with unstressed ones (Figures 3 and 4). Notice that basal values of MAP and HR didn't differ reliably between sexes in NT group (Table 1).

Thus, in NT rats, acute effects of NO blockade with L-NAME on the MAP and HR were more pronounced in female than in male rats at rest and especially during stress.

\subsection{Hypertension Versus Normotension}

Renovascular hypertension was accompanied by a decrease in vascular sensitivity to NO blockade at rest, which was similar in both sexes (Figures 3 and 5). Indeed, the vasoconstrictor response to NO blockade was attenuated in HT rats compared with NT animals of both sexes $(17 \%$ vs. $25 \%, \mathrm{p}<0.05$ for females; $10 \%$ vs. $17 \%$, $\mathrm{p}<0.05$ for males, respectively). However, despite less pronounced pressor reactions after L-NAME injection in HT vs. NT rats, the MAP didn't normalize even at 120 minutes of experiment in female and male HT animals. Unlike vascular reactivity, cardiac responses to L-NAME in NT and HT rats were comparable. As a result, in HT rats as in NT ones, pressor and cardiac effects of LNAME were greater in females than in males $(17 \%$ against $10 \%, \mathrm{p}<0.05$ for MAP; $26 \%$ against $12 \%, \mathrm{p}<$ 0.05 for HR).

Pressure responses to IS against L-NAME compared with IS alone were significantly changed in HT females and slightly changed in HT males (Figures $\mathbf{2}$ and 6). Actually, in HT females, NO blockade induced more significant stress-evoked elevation in MAP than IS alone did $(24 \%$ vs. $12 \%, \mathrm{p}<0,05)$ and abolished completely the long-lasting hypotensive state observed after shortlasting pressor response during IS alone. As for HT males, NO blockade didn't affect the amplitude of MAP response to IS but it increased the duration of stressinduced hypertension. As a result, in contrast to IS alone, IS against NO blockade induced the similar MAP responses pattern in male and female HT rats.

In both male and female HT rats, NO blockade abolished completely the tachycardic effect of IS (Figures 2 and 6). Note, that in HT females, tachycardic effect of IS was replaced by slight non-significant but long-lasting (120 min) bradicardic effect of L-NAME. There were no statistical differences in baseline HR values between HT females and HT males in this experiment (Table 1).

\section{DISCUSSION}

In this study we examined the effect of NO blockade by L-NAME on MAP and HR in conscious NT and HT male and female rats at rest and during IS. The results show that the cardiovascular sensitivity to L-NAME depended upon the following factors: resting MAP (normotension vs. hypertension), gender (male vs. female), and state (rest vs. stress).

We have, for the first time, demonstrated that the severity of hypertension associated with impairment of renal blood flow was lower in females than in males. These findings are in agreement with our previous reports $[15,16]$ and results of other studies $[20,21]$ in which showed the sexual dimorphism in development of several forms of hypertension in both humans and animals.

In addition, we found that both NT and HT females demonstrated more favorable pattern of cardiovascular responses to IS. Actually, in NT females the chronotropic effects of IS were more pronounced, while the 


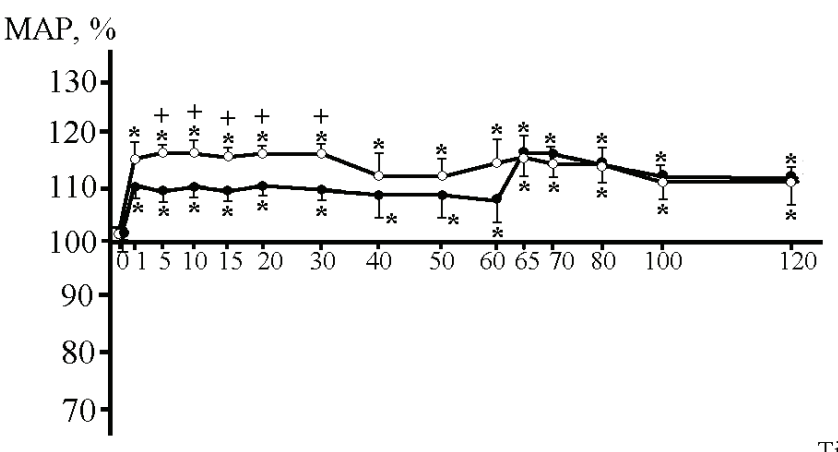

(a)

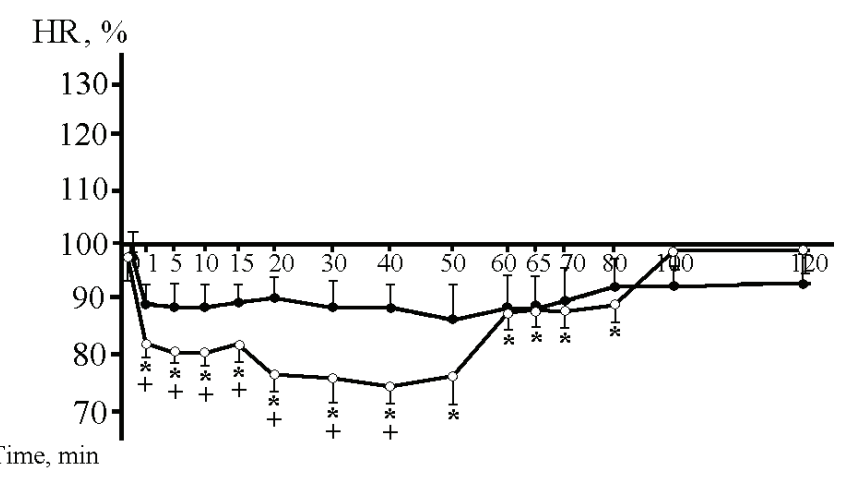

(b)

Figure 5. The effects of NO synthesis inhibition by L-NAME on mean arterial pressure (MAP, \% $-a)$ and heart rate $(\mathrm{HR}, \%-b)$ in hypertensive male and female rats at rest.

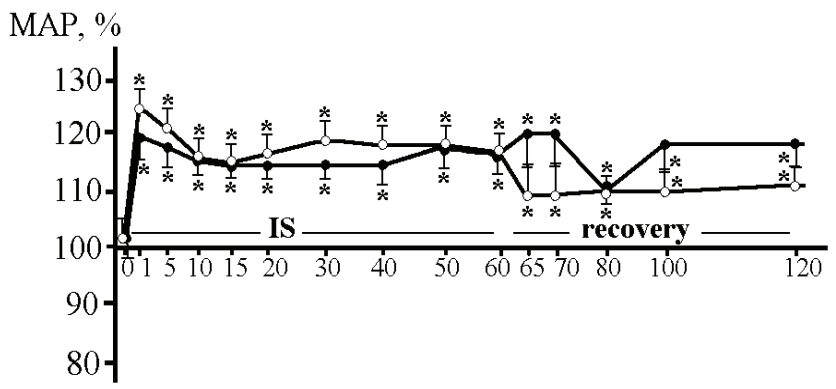

(a)

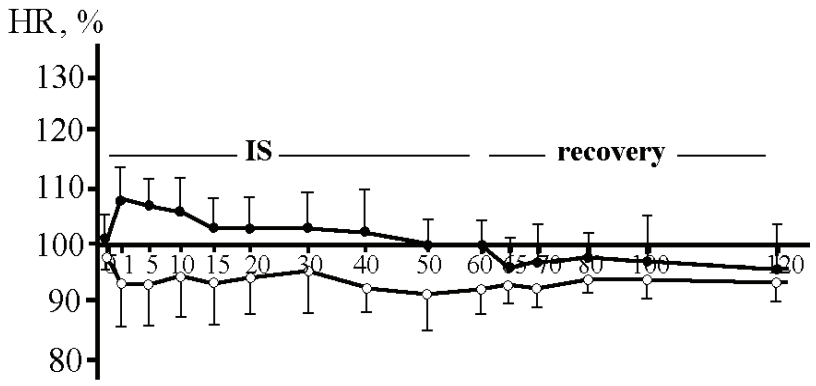

Time, $\min$

(b)

Figure 6. The effects of NO synthesis inhibition by L-NAME on mean arterial pressure (MAP, $\%-a)$ and heart rate (HR, \% - b) in hypertensive male and female rats under stress condition.

pressor effects were weakened compared with males. Hemodynamic parameters in females returned to normal more rapidly than in males. The more pronounced hypertension in males vs. females was associated with increased cardiovascular stress-reactivity in males and with decreased cardiovascular stress-reactivity in females. Moreover, only in NT and HT females shortlasting stress-induced MAP elevation was replaced by transient hypotension indicating the activation of compensatory mechanisms.

In so far as heightened pressure stress responses had been linked to the development of cardiovascular diseases [22], from our result, we concluded that more favorable pattern of cardiovascular responses to stress, observed in NT and especially HT females compared with male groups may be an important mechanism responsible for greater resistance of females to hypertension and other cardiovascular diseases. Indeed, our previous observation demonstrated that NT male vs. NT female rats have been shown to exhibit heightened pressure stress-reactivity and lower cardiovascular and cerebrovascular resistance to stress-induced damages [23, 24].

In order to investigate the role of NO in gender-related differences in the severity of renovascular hypertension and in cardiovascular stress responses in NT and HT rats, we studied the effect of NO blockade on hemodynamic parameters at rest and during stress.

The results of the present study show that in both NT and HT rats, NO blockade by L-NAME caused an increase in MAP with a decrease in HR and these changes depended upon the gender and the state of rats.

At rest, NO blockade increased the MAP and decreased the HR more effectively in female NT and HT rats than in male groups. During stress, NO blockade modified the stress-induced MAP and HR responses more significantly in female NT and HT rats compared with male groups. Our results are consistent with reports of in vitro observations that showed stronger nitrergic control of various vessels of female than in male rats in normotensive state [11-14]. These data, along with report of higher NO production [25] and plasma arginine levels [26] in female gender compared with male gender are in accordance with our previous report of greater blood NO concentration in both NT and HT females vs. male groups under normal and stress conditions [15].

This male-female difference suggesting the more effective NO control of cardiovascular activity in females 
vs. males may be an important mechanism responsible for more favorable stress-induced pattern of MAP and HR changes observed in NT and especially HT females compared with NT and HT males.

In the work of some investigators [6,7] it has been demonstrated that the decrease in NO activity is a negative factor promoting hypertension development and increasing the risk of cardiovascular complications. Indeed, we found that renovascular hypertension being more severe in males than in females was accompanied by a decrease in effectiveness of NO control of MAP at rest and during stress. Moreover, our previous report demonstrated that more pronounced hypertension in male vs. female rats was accompanied by more significant decrease in NO production in males than in females [15]. These findings led to a suggestion that a decrease of function of the NO-ergic system as a major factor contributing to hypertension.

Our results are consistent with reports of diminished availability of basal NO in blood [15], urine [27], kidney [28] and in vessels [29] in hypertensive state. Yet some investigators have not revealed any differences in nitrite/nitrate plasma concentration [30] and hemodynamic responses to NO blockade [31] between NT and HT rats. But others have found enhanced endothelial NO synthase (eNOS) activity in endothelial cells from isolated aorta [32] and elevated hemodynamic responses to NO blockade [33] in HT vs. NT rats, suggesting that NO production is increased rather than decreased in the HT animals. This conclusion seems to contradict the results of the present study. However, the involvement of NO synthesis and release in the hypertensive state remains obscure. The enhanced NO production in the vascular beds in hypertensive state may represent a compensatory mechanism to blood pressure increasing. So, McIntary et al. [32] observed that in both NT and HT male rats compared with females the higher baseline blood pressure was associated with greater eNOS activity in aorta. The increased sympathetic activity being important factor in pathogenesis of hypertension was accompanied by an increase in activity of neuronal isoform of NO synthase that tonically inhibits the sympathetic outflow from the specific area of the brain involved in the neurogenic control of blood pressure [34]. These facts are further evidence that NO production is increased as a compensatory mechanism. It therefore seems likely that the reduced NO availability in hypertensive state observed in many studies is due to increased scavenging. Furthermore, discrepant results among studies may be due to the fact that above experiments was done by topical application of the pharmacological agents which modulate of NO activity in different section of the pial vessels. The results could be different from those ob- tained in the whole vascular beds or in local circulation.

High nitrergic control of cardiovascular activity in females may be due to estrogens that stimulate eNOS function and NO production, potentiate the endothelium-dependent effect of acetylcholine [35]. On the contrary, androgens through a mediated increase in angiotensin II, which causes oxidative stress with enhanced superoxide production, quenching of $\mathrm{NO}$, contribute to decrease in NO availability [20]. In addition, androgens appear to play an inhibitory role in small intestinal endothelial function [36]. The reciprocal stimulatory effects of NO and cholinergic influences being more pronounced in females vs. males [37] may be an additional mechanism responsible for enhanced NO activity in females compared with males. Notice, that the augmented parasympathetic activity has protective effects during myocardial infarction and coronary sudden death [38, 39]. Based on these data one may suggest that specific relationship between NO activity and cholinergic system reduces risk of progressive hypertension and cardiovascular disorders in females.

\section{CONCLUSIONS}

In summary, our results indicate that both NT and HT females demonstrated the more effective NO control of cardiovascular activity under normal and especially stress conditions than male groups. This male-female difference may be important mechanism responsible for greater in females vs. males of cardiovascular resistance to stress and development of hypertension observed in experimental animal models and in humans.

\section{ACKNOWLEDGEMENTS}

This studies were supported in part by a Grant from Russian Ministry of Science and Education within program "Scientific and scientific and pedagogical staff of innovative Russia" 2009-2012, in the field of "Fundamental Medicine and Physiology", GK- 1257P and GK-144P Conflict of Interest. Conflict of Interest: none declared.

\section{REFERENCES}

[1] Beghetti, M., Sparling, C., Cox, P., Stephens, D. and Adatia, I. (2003) Inhaled NO inhibits platelet aggregation and elevates plasma but not intraplatelet cGMP in healthy human volunteers. American Journal Physiology -Heart and Circulatory Physiology, 285(2), H637-642.

[2] Urao, N., Okigaki, M., Yamada, H., Aadachi, Y. Matsuno, K. and Matsui, A. (2006) Erythropoietin-Mobilized Endothelial Progenitors Enhance Reendothelialization via Akt-Endothelial Nitric Oxide Synthase Activation and Prevent Neointimal Hyperplasia. Circulation Research, 98(11), 1405-1411. 
[3] Sener, A. and Smith, F. (2001) Nitric oxide modulates arterial baroreflex control of heart rate in conscious lambs in an age-dependent manner. American Journal Physiology-Heart and Circulatory Physiology, 280(5), H2255-H2263.

[4] Herring, N., Danson, E. and Paterson, D. (2002) Cholinergic control of heart rate by nitric oxide is site specific. News Physiological Sciences, 17(6), 202-206.

[5] Patel, K., Li, Y.-F. and Hirooka, Y. (2001) Role of nitric oxide in central sympathetic outflow. Experimental Biology and Medicine, 226(9), 814-824.

[6] Markov, K. (2001) Nitrogen oxide and the cardiovascular system. Uspechi Fiziologicheskikh Nauk, 32(3), 49-65.

[7] Toda, N. and Okamura, T. (2003) The pharmacology of nitric oxide in the peripheral nervous system of blood vessels. Pharmacological Reviews, 55(2), 271-324.

[8] Ignarro, L., Napoli, C. and Loscalzo, J. (2002) Nitric oxide donors and cardiovascular agents modulating the bioactivity of nitric oxide. Circulation Research, 90(1), 21-28.

[9] Forte, P., Kneale, B., Milne, E., Chowienczyk, P., Johnston, A., Benjamin, N. and Ritter, J. (1998) Evidence for a difference in nitric oxide biosynthesis between healthy women and men. Hypertension, 32(4), 730-734.

[10] Sader, M. and Celermajer, D. (2002) Endothelial function, vascular reactivity and gender differences in the cardiovascular system. Cardiovascular Research, 53(3), 597604.

[11] Kauser, K. and Rubanyi, G. (1994) Gender difference in bioassayable endothelium-derived nitric oxide from isolated rat aortae. American Journal of Physiology - Heart and Circulatory Physiology, 267(5), H2311-2317.

[12] Skarsgard, P., van Breemen, C. and Laher, I. (1997) Estrogen regulates myogenic tone in pressurized cerebral arteries by enhanced basal release of nitric oxide. American Journal of Physiology - Heart and Circulatory Physiology, 273(5), H2248-2256.

[13] Knot, H., Lounsbury, K., Brayden, J. and Nelson, M. (1999) Gender differences in coronary artery diameter reflect changes in both endothelial $\mathrm{Ca}^{2+}$ and ecNOS activity. American Journal of Physiology - Heart and Circulatory Physiology, 276(3), H961- 969.

[14] Laughlin, M., Welshons, W., Sturek, M., Rush, J., Turk, J., Taylor, J., et al. (2003) Gender, exercise training, and eNOS expression in porcine skeletal muscle arteries. Journal of Applied Physiology, 95(1), 250-264.

[15] Glushkovskaya-Semyachkina, O., Anishchenko, T., Sindyakova, T., Leksina, O. and Berdnikova, V. (2006) Sexrelated differences in nitric oxide content in healthy and hypertensive rats at rest and stress conditions. Bulletin of Experimental Biology and Medicine, 142(1), 9-13.

[16] Anishchenko, T., Glushkovskaya-Semyachkina, O., Berdnikova, V. and Sindyakova, T. (2007) Sex-related differences in cardiovascular stress-reactivity in healthy and hypertensive rats. Bulletin of Experimental Biology and Medicine, 143(2), 136-139.

[17] Bondarenko, O., Bondarenko, N., Malyshev, I. and Manukhina, E. (2001) Antistress effect of nitric oxide. Biological Bulletin, 28(3), 387-393.

[18] Dias, A. and Colombari, E. (2006) Central nitric oxide modulates hindquarter vasodilation elicited by AMPA receptor stimulation in the NTS of conscious rats. American Journal of Physiology - Regulatory Integrative Comparative Physiology, 290(5), R1330-R1336.

[19] Anishchenko, T. and Igosheva. (1992) Sex differences in reactivity of conscious and anesthetized rats to surgical stress. Bulletin of Experimental Biology and Medicine, 113(1), 26-28.

[20] Reckelhoff, J.F. (2001) Gender Differences in the Regulation of Blood Pressure. Hypertension, 37(4), 1199- 1223.

[21] Coatmellec-Taglioni, G., Dausse, J., Ribière, C. and Giudicelli, Y. (2003) Sexual Dimorphism in cafeteria dietinduced hypertension is associated with gender-related difference in renal leptin receptor down-regulation. Journal of Pharmacology and Experimental Therapeutics, 305(1), 362-367.

[22] Matthews, K., Katholi, C., McCreath, H., Whooley, M. Williams, D., Zhu, S. and Markovitz, J. (2004) Blood pressure reactivity to psychological stress predicts hypertension in the CARDIA study. Circulation, 110(1), 74 78.

[23] Anishchenko, T., Brill, G., Romanova, T. and Shorina, L. (1995) Sex-related differences in the degree of lipid peroxidation activation and resistance to cardiovascular damage induced by stress in rats. Bulletin of Experimental Biology and Medicine, 119(4), 354-357.

[24] Anishchenko, T., Brill, G., Romanova, T. and Igosheva, N. (1992) Sex differences in adrenocortical sensitivity and resistance to cerebral lesions in rats exposed to severe stress. Bulletin of Experimental Biology and Medicine, 114(10), 353-355.

[25] Forte, P., Kneale, B., Milne, E., Chowienczyk, P., Johnston, A., Benjamin, N. and Ritter, J. (1998) Evidence for a difference in nitric oxide biosynthesis between healthy women and men. Hypertension, 32(4), 730-734.

[26] Luiking, Y., Hallemeesch, M., Vissers, Y., Lamers, W. and Deutz, N. (2004) In vivo whole body and organ arginine metabolism during endotoxemia (sepsis) is dependent on mouse strain and gender. The Journal of $\mathrm{Nu}$ trition, 134(10), 2768S-2774S.

[27] Forte, P., Copland, M., Smith, L., Milne, E., Sutherland, J. and Benjamin, N. (1997) Basal nitric oxide synthesis in essential hypertension. Lancet, 349(3), 837-842.

[28] Tokuyama, H., Hayashi, K., Matsuda, H., Kubota, E., Honda, M. and Ozawa, Y. (2002) Stenosis-dependent role of nitric oxide and prostaglandins in chronic renal ischemia. American Journal of Physiology - Renal Physiology, 282(1), F859-F865.

[29] Rosyn, M., Speziale, E., Celentano, M., Vega, G., Damiano, P. and Puyy, A. (2001) Serotonin hypersensitivity in aorta of two-kidney one clip hypertensive rats: Calcium contribution and prostanoids-nitric oxide interactions. Archives of Physiology and Biochemistry, 109(1), 32-37.

[30] Puyo, A., Vega, G., Iraldi, A., Albornoz, L., Roson, M. and Scaglia, P. (1998) Atrial natriuretic factor in two kidney-two clip renovascular hypertension in the rat. Medicine (Buenos Aires), 58(2), 165-170.

[31] Fozard, J. and Part, M. (1991) Hemodynamic responses to $\mathrm{N}^{\mathrm{G}}$-monomethyl-L-arginine in spontaneously hypertensive and normotensive Wistar-Kyoto rats. British Journal Pharmacology, 102(4), 823-826. 
[32] McIntyre, M., Hamilton, C., Rees, D., Reid, J. and Dominiczak, A. (1997) Sex differences in the abundance of endothelial nitric oxide in a model of genetic hypertension. Hypertension, 30(6), 1517-1524.

[33] Lacolley, P., Lewis, S. and Brody, M. (1991) L-N ${ }^{\mathrm{G}}$ nitroar-ginine produces and exaggerated hypertension in anesthetized SHR. European Journal Pharmacology, 197(2-3), 239- 240.

[34] Campese, V. (2000) The kidney and hypertension: Over 70 years of research. Journal of Nephrology, 19(6), 691698.

[35] Orshal, J. and Khalil, R. (2004) Gender, sex hormones, and vascular tone. American Journal of PhysiologyRegulatory Integrative Comparative Physiology, 286(2), R233-R249.

[36] Ba, Z., Yokoyama, Y., Toth, B., Rue, L., Bland, K. and Chaudry, I. (2004) Gender differences in small intestinal endothelial function: Inhibitory role of androgens. $\mathrm{Am}$ - erican Journal of Physiology-Gastrointestinal Liver Physiology, 286(2), G452-G457.

[37] Anishchenko, T. and Glushkovskaya-Semaychkina, O. (2003) Normalized entropy applied to the analysis of gender-related differences in parasympathetic cardiovascular control in normal and stressed rat. Physician and Technology, 32(1), 29-39.

[38] Airaksinen, K., Ikaheimo, M., Linnaluoto, M., Tahvanainen, K. and Huikuri, H. (1998) Gender difference in autonomic and hemodynamic reactions to abrupt coronary occlusion. Journal of American College of Cardiology, 31(2), 301-306.

[39] De Ferrari, G., Vanoli, E., Stramba-Badiale, M., Hull, S. and Foreman, R. (1991) Vagal reflexes and survival during acute myocardial ischemia in conscious dogs with healed myocardial infarction. American Journal Physiology-Heart and Circulatory Physiology, 261(1), H63H69. 